2 Jakobsen M, Anker N, Dollerup J, et al. Study on drug costs associated with COPD prescription medicine in Denmark. Clin Respir J 2013; 7: 328-337.

Blanchette CM, Dalal AA, Mapel D. Changes in COPD demographics and costs over 20 years. J Med Econ 2012; 15 : 1176-1182.

4 Corsonello A, Pedone C, Corica F, et al. Polypharmacy in elderly patients at discharge from the acute care hospital. Ther Clin Risk Manag 2007; 3: 197-203.

5 Hajjar ER, Hanlon JT, Artz MB, et al. Adverse drug reaction risk factors in older outpatients. Am J Geriatr Pharmacother 2003; 1: 82-89.

6 Gellad WF, Grenard JL, Marcum ZA. A systematic review of barriers to medication adherence in the elderly: looking beyond cost and regimen complexity. Am J Geriatr Pharmacother 2011; 9: 11-23.

7 Hovstadius B, Åstrand B, Persson U, et al. Acquisition cost of dispensed drugs in individuals with multiple medications - a register-based study in Sweden. Health Policy 2011; 101: 153-161.

8 Almagro P, López García F, Cabrera FJ, et al. Comorbidity and gender-related differences in patients hospitalized for COPD. The ECCO study. Respir Med 2010; 104: 253-259.

9 Vestbo J, Hurd SS, Agustí AG, et al. Global strategy for the diagnosis, management, and prevention of chronic obstructive pulmonary disease: GOLD executive summary. Am J Respir Crit Care Med 2013; 187: $347-365$.

10 Hajjar ER, Cafiero AC, Hanlon JT. Polypharmacy in elderly patients. Am J Geriatr Pharmacother 2007; 5: 345-351.

11 Nobili A, Marengoni A, Tettamanti M, et al. Association between clusters of diseases and polypharmacy in hospitalized elderly patients: results from the REPOSI study. Eur J Intern Med 2011; 22: 597-602.

12 Tinetti ME, Bogardus ST Jr, Agostini JV. Potential pitfalls of disease-specific guidelines for patients with multiple conditions. N Engl J Med 2004; 351: 2870-2874.

13 Travers J, Marsh S, Caldwell B, et al. External validity of randomized controlled trials in COPD. Respir Med 2007; 101: 1313-1320.

14 Holmes HM. Rational prescribing for patients with a reduced life expectancy. Clin Pharmacol Ther 2009; 85: $103-107$.

15 Cook DE, Sidel M, Belletti DA, et al. Review: clinical inertia in the management of chronic obstructive pulmonary disease. COPD 2012; 9: 73-80.

\title{
Does a single Pseudomonas aeruginosa isolation predict COPD mortality?
}

To the Editor:

Patients with chronic obstructive pulmonary disease (COPD) often suffer from acute exacerbations (AECOPD) of their disease, which have a significant impact on their health status [1]. Evidence suggests that $\sim 50 \%$ of these exacerbations are attributable to bacteria [2]. Pseudomonas aeruginosa can cause AECOPD and is associated with reduced survival in cystic fibrosis (CF) and bronchiectasis [3, 4]. Some studies have found that the presence of $P$. aeruginosa is also associated with mortality in COPD, but these findings have been based on patients hospitalised with exacerbations [5, 6] or those hospitalised with multidrug-resistant organisms [7].

The impact of $P$. aeruginosa identified in sputum from COPD outpatients is less clear but is an important issue for determining how aggressive strategies to attempt eradication should be. Therefore, we conducted a nested case-control study to investigate whether the isolation of $P$. aeruginosa in the sputum of a general COPD population was associated with long-term mortality.

We first identified all sputum specimen results from Royal Brompton Hospital (London, UK) microbiology records between 2000 and 2012. These were cross-correlated with patients listed on our COPD research audit database. The reason for obtaining a sputum culture was not recorded systematically, but included repeated exacerbations and deterioration in symptom severity and/or clinical status, as well as opportunistic collection from patients with chronic sputum production. The laboratory threshold for a culture to be considered P. aeruginosa positive was $200 \mathrm{CFU} \cdot \mathrm{mL}^{-1}$ (a semi-quantitative method). For patients with repeated positive sputum cultures the date of the first culture was recorded. Demographic variables, lung function measurements, gas transfer data, arterial blood gases and exacerbation frequency during the year prior to entering the study were recorded for all patients.

All analyses were performed using the Predictive Analytics Software (version 18; SPSS Inc., Chicago, IL, USA). Group comparisons were conducted utilising a t-test or the Chi-squared test as appropriate. Proportional Cox hazard analysis was utilised to assess: 1) the impact of parameters that differed between $P$. aeruginosa culture-positive and culture-negative groups and 2) the impact of antibiotic treatment on mortality. The proportionality hazard assumption was tested using partial residual plots (Schoenberg 
TABLE 1 Baseline differences between the initial cohort and the study population, and the Pseudomonas aeruginosa culturepositive and culture-negative groups

Initial cohort

Study population

p-value ${ }^{\# \quad P . ~ a e r u g i n o s a ~ c u l t u r e-~}$ positive group
P. aeruginosa culture- $\quad$-value ${ }^{\pi}$ negative group

\begin{tabular}{|c|c|c|c|c|c|c|}
\hline Subjects $n$ & 380 & 132 & & 66 & 66 & \\
\hline Age years & $65.4 \pm 10.9$ & $68 \pm 8.8$ & 0.011 & $68 \pm 8.8$ & $68.3 \pm 9$ & 0.969 \\
\hline \multicolumn{7}{|l|}{ Sex $\%$} \\
\hline Male & & & & 51.1 & 51.1 & 0.999 \\
\hline BMI $\mathbf{m} \cdot \mathrm{kg}^{-2}$ & $24.7 \pm 5.2$ & $24 \pm 6.3$ & 0.257 & $23.6 \pm 5.8$ & $24.4 \pm 6.8$ & 0.497 \\
\hline FEV $1 \%$ predicted & $38.6 \pm 20.1(9-81.1)$ & $33.6 \pm 14.7(12.6-72.4)$ & 0.008 & $33.4 \pm 14.5(12.6-72.4)$ & $33.8 \pm 14.9(12.9-71)$ & 0.882 \\
\hline FEV $1 /$ FVC $\%$ & $40.6 \pm 21.2(13-69.8)$ & $36.1 \pm 16.8(13.5-69.8)$ & 0.029 & $35 \pm 12.1(14.1-69.8)$ & $37.2 \pm 20.7(13.5-69.5)$ & 0.456 \\
\hline TLC $\%$ predicted & $123.7 \pm 19.6$ & $122.7 \pm 17$ & 0.662 & $121.1 \pm 18.1$ & $124.5 \pm 15.6$ & 0.286 \\
\hline RV \% predicted & $205.1 \pm 61.9$ & $203.3 \pm 53$ & 0.780 & $206 \pm 52.8$ & $200.5 \pm 53.6$ & 0.569 \\
\hline $\mathrm{RV} / \mathrm{TLC}$ & $155.2 \pm 32.7$ & $157 \pm 32.1$ & 0.639 & $60.3 \pm 9.8$ & $59.4 \pm 11.6$ & 0.647 \\
\hline $\mathrm{PaO}_{2} \mathrm{kPa}$ & $9.5 \pm 1.4$ & $9.0 \pm 1.5$ & 0.014 & $9.1 \pm 1.4$ & $8.9 \pm 1.8$ & 0.440 \\
\hline $\mathrm{PaCO}_{2} \mathrm{kPa}$ & $5.3 \pm 0.8$ & $5.5 \pm 1.1$ & 0.116 & $5.3 \pm 0.9$ & $5.8 \pm 1.4$ & 0.018 \\
\hline Exacerbations per year & $1.9 \pm 0.7$ & $2.1 \pm 0.7$ & 0.086 & $2.3 \pm 0.7$ & $1.9 \pm 0.7$ & 0.008 \\
\hline
\end{tabular}

Data are presented as mean \pm SD or mean \pm SD (range), unless otherwise stated. Bold signifies statistical significance. BMI: body mass index; FEV1: forced expiratory volume in $1 \mathrm{~s}$; FVC: forced vital capacity; DLCO: diffusing capacity of the lung for carbon monoxide; Kco: transfer coefficient of the lung for carbon monoxide; TLC: total lung capacity; RV: residual volume; $\mathrm{PaO}_{2}$ : arterial oxygen tension; $\mathrm{PaCO}_{2}$ : arterial carbon dioxide tension. \#: level of significance for comparison between the initial cohort and the study population; ": level of significance for comparison between the $P$. aeruginosa culture-positive and culture-negative groups.

residuals proportionality hazard test). The Kaplan-Meier survival curve with the Log rank comparison was used to assess the impact of $P$. aeruginosa isolation on mortality and estimate the median survival of the $P$. aeruginosa culture-positive and culture-negative groups. A p-value $<0.05$ was considered significant.

The initial cohort consisted of 380 subjects (247 male and 133 female), with a mean \pm SD age of $66.3 \pm 10.3$ years and a forced expiratory volume in $1 \mathrm{~s}(\mathrm{FEV} 1)$ of $36.7 \pm 18.3 \%$ predicted. $95(25 \%)$ patients from the initial population had at least one positive sputum culture for $P$. aeruginosa, while the rest had either negative cultures or cultured bacteria other than P. aeruginosa. 66 P. aeruginosa culture-positive patients from the initial cohort were matched to another 66 P. aeruginosa culture-negative patients for sex, age and $\mathrm{FEV}_{1} \%$ predicted.

Differences in demographics and clinical characteristics between the initial COPD cohort and the study population, and $P$. aeruginosa culture-positive and culture-negative patients are presented in table 1 . Although the initial matching was done according to three baseline variables, the two final groups were found to be similar in all recorded parameters of lung function (forced vital capacity, FEV1/forced vital capacity, diffusing capacity of the lung for carbon monoxide, transfer coefficient of the lung for carbon monoxide, total lung capacity, residual volume and residual volume/total lung capacity), as well as body mass index and arterial oxygen tension. Arterial carbon dioxide tension $(p=0.018)$ and exacerbation rate $(\mathrm{p}=0.008)$ were higher in the $P$. aeruginosa culture-positive patients compared to the $P$. aeruginosa culturenegative patients (table 1). However, proportional Cox hazard analysis, which was conducted separately for each group, indicated that exacerbation rate had no impact on mortality ( $P$. aeruginosa culture-positive: hazard ratio 1.334 (95\% CI 0.351-5.067), $\mathrm{p}=0.672$; $P$. aeruginosa culture-negative: hazard ratio $2.161(95 \%$ CI 0.576-8.112), $\mathrm{p}=0.253$ ). The results were similar for arterial carbon dioxide tension ( $P$. aeruginosa culturepositive: hazard ratio 1.134 (95\% CI 0.758-1.697), $\mathrm{p}=0.539$; $P$. aeruginosa culture-negative: hazard ratio 1.074 (95\% CI 0.842-1.369), $\mathrm{p}=0.572$ ), indicating that it was not associated with survival.

Median (range) survival for the study population $(n=132)$ was $81.2(50.8-109.5)$ months. During this period $51(38.6 \%)$ patients died, of these $52.9 \%(n=27)$ were P. aeruginosa culture-positive and $47.1 \%$ $(n=24)$ were $P$. aeruginosa culture-negative. The Kaplan-Meier survival curve, using the Log rank comparison, indicated that $P$. aeruginosa positive sputum culture was not associated with mortality in this population. Median (range) survival for P. aeruginosa culture-positive patients was $80.1(40.1-120.1)$ months compared to $88.6(52-125.3)$ months in the $P$. aeruginosa culture-negative patients $(\mathrm{p}=0.49)$ (fig. 1). Furthermore, no differences in survival as a function of the presence of Pseudomonas would have been identified if the entire population of 380 had been analysed (data not shown). 


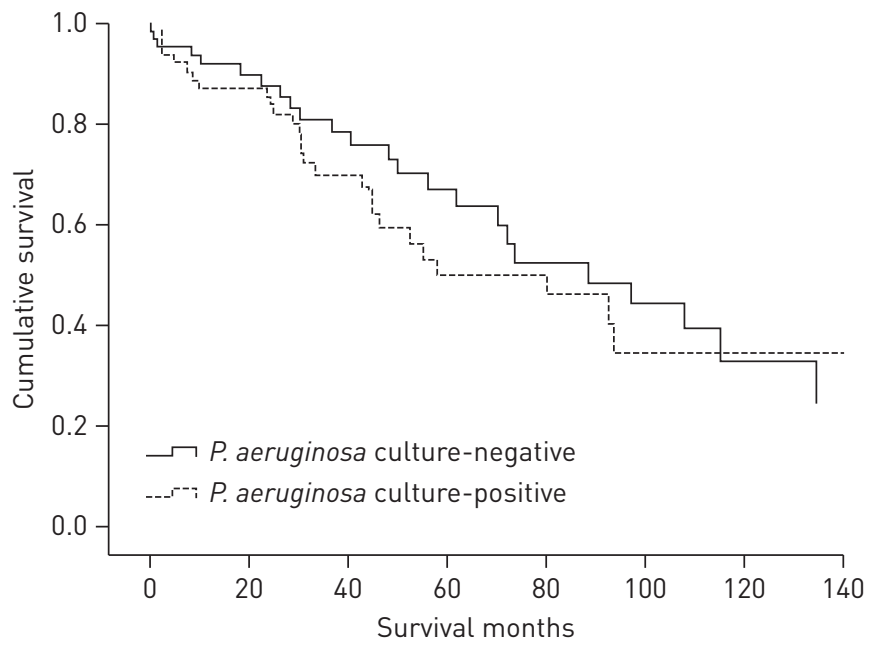

FIGURE 1 Kaplan-Meier survival curve for the Pseudomonas aeruginosa culture-positive and $P$. aeruginosa culture-negative groups. $\mathrm{p}=0.49$ for $P$. aeruginosa culture-negative patients.

A secondary analysis was also undertaken to identify whether Pseudomonas eradication treatment among $P$. aeruginosa culture-positive patients had any impact on survival. Sputum samples were ordered on a clinical basis and the decision of whether to attempt to eradicate Pseudomonas was at the discretion of the treating physician. Out of 66 P. aeruginosa culture-positive patients, $34(25.8 \%)$ did not receive any treatment while $32(24.2 \%)$ were treated either with oral ciprofloxacin or appropriate intravenous antibiotics, depending on resistance pattern. These two patient subgroups were similar regarding age, sex, body mass index, pulmonary function testing variables and exacerbation rate (data not shown). Cox proportional hazard analysis indicated that $P$. aeruginosa treatment was not associated with mortality (hazard ratio $1.452(95 \%$ CI 0.666-3.163), $\mathrm{p}=0.348$ ) among $P$. aeruginosa culture-positive patients.

This study indicates that a single isolate of $P$. aeruginosa in sputum from a general COPD population is not associated with worse survival. Recently, three different patterns of $P$. aeruginosa infection in COPD patients have been described: 1) short carriage and clearance of a P. aeruginosa strain; 2) acquisition of a new strain associated with an AECOPD; and 3) persistence of $P$. aeruginosa colonisation with unknown clinical significance [8]. Although the specific pattern of $P$. aeruginosa infection was not investigated in the current study, our results indicate that even though a single positive $P$. aeruginosa sputum culture was correlated with a higher exacerbation frequency, it was not associated with a worse long-term outcome in COPD outpatients.

The results of our study may, to some extent, be limited by its retrospective design and by the relatively small number of patients included, although a formal power estimation was not conducted. However, most of the published studies in the field have used similar sample sizes to investigate the potential impact of $P$. aeruginosa infection on COPD survival $[2,6,9]$. Another limitation is the lack of baseline data on exercise capacity, dyspnoea severity and frequency of previous hospitalisations, all of which could have affected mortality. Moreover, high-resolution computed tomography of the chest was not systematically conducted in all patients so the prevalence of bronchiectasis, a factor which could have favoured P. aeruginosa infection, could not be estimated; although it should be noted that our institution runs separate clinics for patients known to have either CF or non-CF bronchiectasis. However, several of these prognostic factors $[6,7]$ and imaging data $[6,7,9]$ are also lacking in previously published studies that investigated the effect of $P$. aeruginosa on COPD mortality. Furthermore, careful patient matching has controlled for most of the lung function parameters that could affect mortality [10], so these negative results could not be attributed to a selected patient subgroup.

In conclusion, this study indicated that a single $P$. aeruginosa sputum isolation is not a predictor of longterm mortality in a general COPD outpatient population. Current clinical practice usually targets $P$. aeruginosa eradication using radical treatment, after its isolation in the sputum of a COPD patient with clinical deterioration or frequent exacerbations. Although eradicating Pseudomonas may be justified to improve health status, patients and clinicians can, to an extent, be reassured by these data that if this is not possible the impact on survival of a single P. aeruginosa isolation is not as significant as it is in CF [3] or bronchiectasis [4]. Future prospective, case-control studies are needed in order to define exact criteria for chronic $P$. aeruginosa infection in COPD patients and the best stratification criteria for a clinical trial of $P$. aeruginosa eradication in COPD. 
ERSpublications

A single positive Pseudomonas aeruginosa sputum culture is not associated with increased longterm mortality in COPD http://ow.ly/x5wvD

Afroditi K. Boutou ${ }^{1}$, Yogini Raste ${ }^{1}$, Jeremy Reid ${ }^{1}$, Khalid Alshafi ${ }^{2}$, Michael I. Polkey ${ }^{1}$ and Nicholas S. Hopkinson ${ }^{1}$ ${ }^{1}$ NIHR Respiratory Biomedical Research Unit, Royal Brompton and Harefield NHS Foundation Trust and Imperial College, London, UK. ${ }^{2}$ Dept of Microbiology, Royal Brompton Hospital, London, UK.

Correspondence: Afroditi K. Boutou, 9-13, Stratigou Sarafi Str, Kalamaria, 55132 Thessaloniki, Greece.

E-mail: afboutou@yahoo.com

Received: Dec 102013 | Accepted after revision: May 132014 | First published online: July 172014

Support statement: This study was supported by the NIHR Respiratory Biomedical Research Unit (Royal Brompton and Harefield NHS Foundation Trust and Imperial College, London, UK).

Conflict of interest: None declared.

\title{
References
}

1 Kelly JL, Bamsey O, Smith C, et al. Health status assessment in routine clinical practice: the chronic obstructive pulmonary disease assessment test score in outpatients. Respiration 2012; 84: 193-199.

2 Wilson R, Sethi S, Anzueto A, et al. Antibiotics for treatment and prevention of exacerbations of chronic obstructive pulmonary disease. J Infect 2013; 67: 497-515.

3 Pressler T, Bohmova C, Conway S, et al. Chronic Pseudomonas aeruginosa infection definition: EuroCareCF Working Group report. J Cyst Fibros 2011; 10: Suppl. 2, S75-S78.

4 Loebinger MR, Wells AU, Hansell DM, et al. Mortality in bronchiectasis: a long-term study assessing the factors influencing survival. Eur Respir J 2009; 34: 843-849.

5 Almagro P, Salvadó M, Garcia-Vidal C, et al. Pseudomonas aeruginosa and mortality after hospital admission for chronic obstructive pulmonary disease. Respiration 2012; 84: 36-43.

6 Lin SH, Kuo PH, Hsueh PR, et al. Sputum bacteriology in hospitalized patients with acute exacerbation of chronic obstructive pulmonary disease in Taiwan with an emphasis on Klebsiella pneumoniae and Pseudomonas aeruginosa. Respirology 2007; 12: 81-87.

7 Montero M, Domínguez M, Orozco-Levi M, et al. Mortality of COPD patients infected with multi-resistant Pseudomonas aeruginosa: a case and control study. Infection 2009; 37: 16-19.

8 Murphy TF. The many faces of Pseudomonas aeruginosa in chronic obstructive pulmonary disease. Clin Infect Dis 2008; 1534-1535.

9 Renom F, Yáñez A, Garau M, et al. Prognosis of COPD patients requiring frequent hospitalization: role of airway infection. Respir Med 2010; 104: 840-848.

10 Boutou AK, Shrikrishna D, Tanner RJ, et al. Lung function indices for predicting mortality in COPD. Eur Respir J 2013; 42: 616-625.

\section{Does traffic noise influence respiratory mortality?}

\author{
To the Editor:
}

Over the last decade, several studies have investigated the association between noise levels, primarily due to road traffic in large cities, and pathologies not related to either traditional hearing impairments or sleep disorders. These pathologies are mainly hypertension and cardiovascular, connective system and respiratory diseases [1]. Their impact on public health has been assessed in time series studies, indicating a traffic noise effect on both cardiovascular and respiratory hospital admissions rates similar to that attributed to air pollutants [2]. In addition, a recent study has shown an association of noise levels with cardiovascular mortality [3]. The description of the physiopathological mechanisms involved in this association reveal an actual impact of current road traffic noise levels on health [1]. Others question this because of the high correlation between traffic noise and air pollution [4], although a previous study in our setting showed an independent association between noise and cardiovascular mortality from the effect of the primary chemical air pollutants [5]. However, the effect of noise on respiratory mortality has not yet been investigated.

In this study, we examined the association between daily mortality due to respiratory causes (International Classification of Diseases, 9th revision, codes 460-519) and daytime noise levels in the city of Madrid 